\title{
Initial assessment and outcome of head injured patients transferred to a regional neurosurgical service: what do we miss?
}

\author{
Patrick T Grant, Sarah Shrouder
}

\begin{abstract}
Objective-To assess the level of missed extracranial injuries in patients transferred to a regional neurosurgical service for ongoing head injury management.

Methods-A three year prospective study conducted under the auspices of the Scottish Trauma Audit Group. All patients were followed during their hospital stay by independent audit staff, their injuries being recorded and scored using established criteria.

Results-115 head trauma patients were transferred during the study period. 15 patients died (13\% mortality). Eight of a total of 87 separate, scorable extracranial injuries were missed (error rate $9 \%$ ), none of which was serious. There were no missed injuries in patients who died. $77 \%$ of patients were managed by an accident and emergency doctor of at least registrar grade.

Conclusions-In contrast to other published series, this study has shown a low rate of missed extracranial injuries in a group of patients whose initial assessment is notoriously difficult. Early involvement by an experienced accident and emergency doctor may play an important part in the overall management of such patients.
\end{abstract}

$(\Im$ Accid Emerg Med 1997;14:10-12)

Keywords : head injury transfer; missed injuries; neurosurgery

Accident and

Emergency

Department, Western

Infirmary, Glasgow

G11 6NT

P T Grant

Scottish Trauma Audit Group

S Shrouder

Correspondence to:

Mr P T Grant, consultant in accident and emergency medicine.

Accepted for publication 7 August 1996 altered mental status, whether this results head injury, drugs, or alcohol. In addition, optimising this initial assessment is particularly important in those patients who are subse-

Diagnostic accuracy has, not surprisingly,
Initial assessment of seriously injured patients quently transferred to another hospital facility for further management.

We therefore decided specifically to analyse all head injured patients transferred from our hospital to the regional neurosurgical service at the Southern General Hospital, Glasgow, during a three year time period. The aim of this study was to determine our level of missed extracranial injury in those patients whom we transferred for ongoing head injury management, and to ascertain what, if any, effect such missed injuries had on patients' survival.

\section{Methods}

This prospective study was conducted under the auspices of the Scottish Trauma Audit Group (STAG). STAG is a centrally funded organisation the aims and objectives of which are to improve the overall management of the seriously injured in Scotland. Currently, six teaching hospitals nationally are contributing to our database. The patients are identified for the study in the A\&E department by nursing staff, who record basic physiological variables (heart rate, blood pressure, respiratory rate, and Glasgow coma scale) on a standard proforma. Thereafter, all patients are followed up during their hospital stay by independent audit staff-both in our hospital and at the Institute of Neurological Sciences - who document all subsequently identified injuries and major interventions. Patients are followed up until discharge, death, or an in-hospital stay of three months. Accurate necropsy data are obtained on all patients who die within this time period. Patients' injuries are scored centrally for all hospitals by one independent assessor using the abbreviated injury scale (1990, revision). ${ }^{2}$ Using a combination of this anatomical scoring and physiological variables identified on admission (that is, the first recorded observations, before any clinical intervention) the probability of survival can be calculated using standard TRISS methodology which has been clearly described elsewhere. ${ }^{3}$

All STAG participating hospitals contribute to the major trauma outcome study (MTOS UK) based in Manchester, and preliminary findings of our group have recently been reported. ${ }^{4}$ 
This study covered all head injured patients who were admitted to the A\&E department of the Western Infirmary, Glasgow, and thereafter transferred to our regional neurosurgical centre (approximately three miles away) during the three year period from 1 February 1992 until 31 January 1995. The department is staffed medically by two consultants, usually four middle grade staff, six senior house officers, and part time clinical assistants. With regard to head trauma, written departmental policy states that patients with an initial Glasgow coma scale (GCS) score of less than 13 should be treated in our resuscitation room, thus mandating review by an $\mathrm{A} \& \mathrm{E}$ doctor of at least registrar grade. Data were collected on all injuries sustained by our patient group and for those with extracranial injuries the relevant $A \& E$ record card was obtained and reexamined. We considered any injury which was not documented on this card to have been "missed". When assessing missed injuries, all fractures or joint injuries had to be confirmed radiologically and the soft tissue component of an associated fracture was excluded (for example, retroperitoneal haematoma from a pelvic fracture, subsequent lung contusion with rib fractures, etc). All external soft tissue injuries with an abbreviated injury score (AIS) of 1 were, similarly, excluded. Children aged less than 13 were not included. To ensure $100 \%$ data capture, staff regularly checked hospital admission lists and the corresponding neurosurgical unit's discharge summaries.

\section{Results}

During this three year period approximately 165000 new patients attended the $A \& E$ department, 129 of whom were initially included in the audit. However, seven of these were subsequently excluded as they had isolated spinal cord injuries (with no head injury) and a further seven were shown to have non-traumatic conditions, for example spontaneous intracerebral and subarachnoid haemorrhage. This left a total of 115 patients available for analysis. There were 92 males and 23 females (male to female ratio $4: 1$ ), with a mean age of 38 years (range 13-87). Ten patients did not have significant head injuries (AIS Head = 0 ) and such patients principally had a depressed level of consciousness due to excess alcohol. The median injury severity score (ISS) was 16 (range 1-51). Figure 1 shows ISS breakdown and outcome. Figure 2 shows outcome with respect to initial Glasgow coma scale scores. Overall, 15 (13\%) patients died, all of whom were severely injured (ISS > 15). Median ISS for patients who died was 26 (range 17-45). All patients who died had severe head injuries (13 with AIS Head $=5$, and two with AIS Head = 4) and only two of them had a craniotomy. Eighty four patients $(73 \%)$ had an isolated head injury, 31 of whom had a neurosurgical operation (37\%).

In total $77 \%$ of patients were managed by an $\mathrm{A} \& \mathrm{E}$ doctor of at least registrar grade, and $32 \%$ of the seriously injured (ISS $>15$ ) were managed by a consultant from the $A \& E$ department.

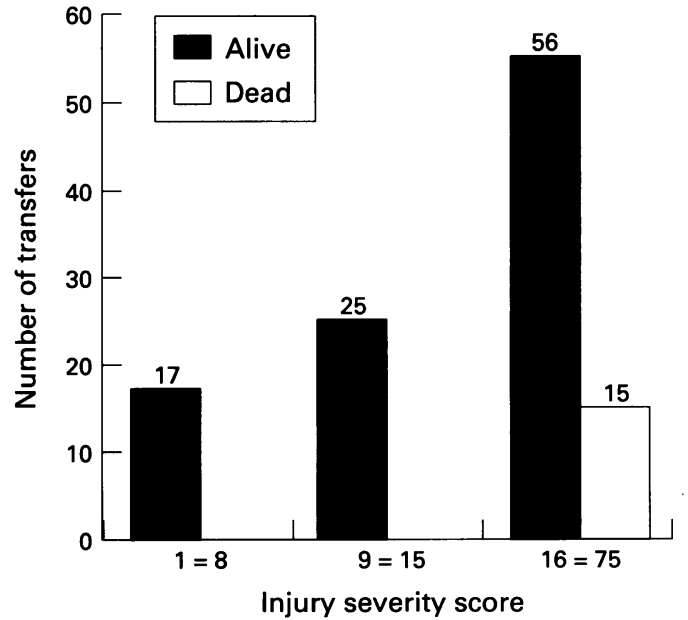

Figure 1 Neurosurgical transfers.

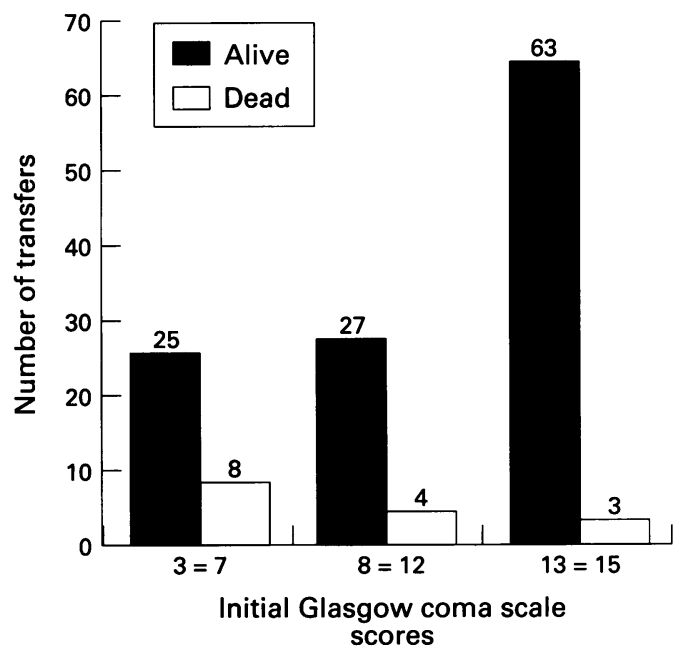

Figure 2 Patient outcome and initial Glasgow coma scale scores.

Table 1 Missed injuries

\begin{tabular}{llrrl}
\hline Age & Sex & GCS & ISS & Missed injury \\
\hline 27 & M & 14 & 21 & Clavicle \\
22 & M & 5 & 8 & Maxilla \\
21 & M & 6 & 29 & Maxilla \\
17 & F & 8 & 29 & Maxilla \\
24 & M & 14 & 19 & Orbit \\
39 & M & 4 & 27 & Crush T3/4 \\
13 & F & 14 & 22 & Zygoma/orbit \\
\hline
\end{tabular}

MISSED INJURIES

In 31 patients, 87 separate scorable extracranial injuries were identified. There were eight missed injuries in seven patients (table 1), giving an overall error rate of $9 \%$.

Most missed injuries were fractures of the face or shoulder girdle, and knowing about them in the $A \& E$ department would not have altered initial management at all. The most potentially serious missed injury occurred in a 39 year old male patient whose dorsal spine fractures were missed after he had fallen from a third floor window. He was shocked on admission (systolic blood pressure $84 \mathrm{~mm} \mathrm{Hg}$ ) and was promptly paralysed and ventilated in the resuscitation room before transfer. No $x$ rays of his dorsal spine were taken at that stage. He made an excellent recovery from his injuries 
Table 2 Delayed transfer deaths

\begin{tabular}{llllll}
\hline Age & GCS & ISS & PS & AIS & Patient details \\
\hline 67 & 15 & 25 & 0.74 & 5 & Occipital skull fracture. Deteriorated 12 h post admission; subdural, no surgery; died day 4. \\
74 & 15 & 26 & 0.72 & 5 & Closed injury, warfarin therapy. INR therapeutic. Deteriorated 6 h later; subdural, no surgery; died day 2. \\
44 & 4 & 26 & 0.73 & 5 & Found in street, ventilated, massive subdural, transferred from ITU; no surgery; died day 28. \\
\hline
\end{tabular}

GCS, Glasgow coma score; ISS, injury severity score; Ps, probability of survival; AIS, abbreviated injury score; INR, international normalised ratio.

and was discharged home, 32 days after admission, with no neurological deficit.

There were no missed injuries in patients who died.

\section{DELAYED TRANSFERS}

Ninety six of our study group were transferred directly to neurosurgical care from the $A \& E$ department. The remaining 19 were initially admitted to an observation ward, intensive care unit, or general surgical operating suite. Of this delayed transfer group one patient had a missed facial bone fracture and three patients subsequently died. Table 2 gives further details about the patients who died, including their probability of survival (Ps).

\section{Discussion}

During the last 20 years numerous studies have highlighted the deficiencies related to the transfers of neurosurgical patients, particularly in relation to adequacy of airway protection and cerebral perfusion. ${ }^{56}$ This, however, was not a major aspect of our study as the Western Infirmary, Glasgow, has its own dedicated "shock team" transfer system, whose activities have previously been reported. ${ }^{7}$ This team would effect the transfer of all multiply injured patients in addition to those with isolated head trauma whose condition may require active airway intervention. It is interesting to note that $73 \%$ of the patients we analysed had an isolated head injury, which is in keeping with other series. Our hospital recently purchased a spiral computerised tomography scanner which is now located adjacent to the $A \& E$ department. Inevitably we are already scanning more "heads" on site, usually at the behest of the receiving neurosurgical team and we therefore expect a reduction in the overall number of patients subsequently transferred to the neurosurgical unit directly from the $A \& E$ department for emergency investigation.

This study has shown the lowest levels of missed extracranial injury yet published either in the United Kingdom or abroad. ${ }^{189}$ It is important to note that none of these injuries threatened life or limb, in comparison with other studies, ${ }^{10-12}$ but-not surprisingly-the most commonly missed extracranial injury was related to the face. The detection of facial bone fractures is obviously important and in most circumstances is done, initially, clinically. Radiography of the facial skeleton is not a high priority in the early management of the severely injured and it may well be that some of these missed fractures were, indeed, suspected clinically, but this was not documented on the A\&E record card.

It is our contention that the A\&E management of the three patients from the "delayed transfer group" who died (table 2) could not be faulted. The most significant missed injury in our study was in a male patient with two crushed thoracic vertebrae. Clearly, the initial detection of such injuries in obtunded patients is very difficult. All our patients were managed according to standard advanced trauma life support (ATLS) guidelines. ${ }^{13}$ However, no specific recommendations are made in this system relating to routine $x$ rays of the dorsal spine in major trauma. We would, nevertheless, agree that victims of blunt trauma related to road traffic accidents or falls, who cannot be assessed clinically, should undergo routine thoracolumbar radiography as soon as is practicable. ${ }^{14}$

For the seriously injured (ISS > 15) overall mortality was $21 \%$ which, again, compares very favourably with recently published series. ${ }^{15}$ Clearly, there is no room for complacency with such a group of compromised patients and we feel that our relatively good results are, at least in part, due to the initial assessment by an experienced $\mathrm{A} \& \mathrm{E}$ doctor interacting with other specialties at an early stage. Continuing efforts are being made to improve senior medical input in the management of these patients and recent STAG data reflect this (D Beard, personal communication).

Regular audit of quality of care for head injured patients is required at every stage in their management if we are going to reduce their overall morbidity and mortality.

We wish to thank the staff of our accident and emergency department for their assistance in collecting data, $\mathrm{Mr} \mathrm{R}$ Johnston, consultant neurosurgeon, for permission to report on his patients, and Miss L Russell for typing the manuscript. The Scottish Trauma Audit Group is funded by a grant from the Clinical Resource and Audit Group of the Scottish Office Home and Health Department.

1 Enderson BL, Reath DB, Meadors J, Dallas W, De Boo J, Maull K. The Tertiary Trauma Survey: a prospective study of missed injury. J Trauma 1990;30:666-9.

2 Joint Committee on Injury Scaling. The abbreviated injury scale: 1990 revision. Illinois: The American Association for scale: 1990 revision. Illinois: The American Associatic

3 Boyd CR, Tolson MA, Copes WS. Evaluating trauma care: the TRISS method. J Trauma 1987;16:882-5.

4 Little K, Steedman DJ, Beard D, Grant P, Crawford R, Ritchie $\mathrm{D}$, et al. Preliminary analysis of the care of injured patients in five Scottish teaching hospitals: first report from the Scottish Trauma Audit Group (STAG). Health Bull 1995;53:55-65.

5 Gentleman D, Jennett B. Hazards of interhospital transfers of comatose head-injured patients. Lancet $1981 ; \mathrm{ii} ; 853-5$.

6 Gentleman D, Jennett B. Audit of transfer of unconscious patients to a neurosurgical unit. Lancet 1990;335:380-4.

7 Reeve W, Runcie CJ, Reidy J, Wallace PGM. Current practice in transferring critically ill patients among hospitals in the west of Scotland. BMJ 1990;300:85-7.

8 Tulloh BR. Diagnostic accuracy in head injured patients: an

9 Lambert SM, Willett K. Transfer of multiply injured patients for neurosurgical opinion: a study of the adequacy

of assessment and resuscitation. Injury 1993;24:333-6. Butterworth JF, Maull KI, Miller JD, Becker DP. Detection
of occult abdominal trauma in patients with severe head of occult abdominal trauma in

11 Gilroy D. Deaths from blunt trauma; a review of 105 cases. Injury 1984;15:304-8.

12 Garland DE, Bailey S. Undetected injuries in head injured adults. Clin Orthop 1981;155:162-5.

13 American College of Surgeons. Advanced trauma life support course. Chicago, Illinois.

14 Terregino CA, Ross SE, Liprinski MF, Foreman J, Hughes $R$. Selective indications for thoracic and lumbar radiography in blunt trauma. Ann Emerg Med 1995;26:126-9.

15 Cooke RS, McNicholl BP, Byrnes DP. Early management of severe head injury in Northern Ireland. Injury 1995;26: 395-7. 